\title{
Simplificación del proceso complejo de reducción de escala de los modelos climáticos globales por medio de la aplicación web SDW
}

\author{
Ronald Gualán $^{1}$, Esteban Sanchez ${ }^{1,2}$, Lenin Campozano ${ }^{1,2}$, Esteban Samaniego ${ }^{1,2}$, Ángel Vazquez ${ }^{1}$ \\ ${ }^{1}$ Departamento de Recursos Hídricos y Ciencias Ambientales, Dirección de Investigación (DIUC) \\ Universidad de Cuenca, Av. Víctor Manuel Albornoz, Campus Quinta Balzaín, Cuenca, Ecuador. \\ ${ }^{2}$ Facultad de Ingeniería, Universidad de Cuenca.
}

Autor para correspondencia: lenin_camp@yahoo.com

Fecha de recepción: 1 de septiembre 2014 - Fecha de aceptación: 15 de noviembre 2014

\section{RESUMEN}

La generación de escenarios de cambio climático está basada en los resultados de los modelos de clima (GCM), los cuales debido su baja resolución requieren la utilización de técnicas de reducción de escala para su aplicación en estudios del impacto del cambio climático a nivel local. De las técnicas de reducción de escala dinámicas, basadas en la resolución de modelos regionales de clima, y las estadísticas, que buscan relaciones estadísticas entre variables sinópticas de clima y observaciones de superficie, las últimas han mostrado una mejor representación de las variables climáticas en zonas de montaña, como es el caso de los Andes. Sin embargo, para considerar las incertidumbres de escenarios de clima futuros entre modelos GCM, entre escenarios de emisiones de gases de efecto invernadero, y entre técnicas de reducción de escala, surge la necesidad del desarrollo de una herramienta versátil que sea parte fundamental de un sistema de soporte de decisiones. Para lograr esto, en el presente trabajo se muestra el desarrollo, implementación y uso de una aplicación web que integra todos los procesos involucrados en la reducción de escala, desde la gestión, análisis y almacenamiento de datos, y generación de modelos, hasta llegar a la entrega de resultados. El sistema presentado podría ser una parte fundamental dentro de un sistema de soporte de decisiones para la adaptación al cambio climático.

Palabras clave: Modelos globales de clima, downscaling, cambio climático, automatización de procesos, aplicación web.

\begin{abstract}
The generation of climate change scenarios is based on the results of global climate models (GCM), which because of their low resolution require the use of downscaling techniques to assess the impact at local scale. Of the downscaling techniques, based either on the use of regional climate models or statistical methods defining the statistical relationship between synoptic climate variables and observations, previous studies revealed that statistical approaches most accurately predict future climate variables in mountainous areas, such as the Andes mountain range. To standardize and facilitate the assessment of the uncertainty between GCM models of future climate, greenhouse gas scenarios, and downscaling techniques, a versatile tool would be most appropriate and helpful. This paper presents the development, implementation and use of a web application that integrates all the steps in downscaling, it is from the search, analysis and storage of data, the selection, calibration and validation of models, up to downscaled outputs. The presented tool could easily be linked to a decision support system for climate change adaptation.
\end{abstract}

Keywords: Global climate models, downscaling, climate change, automation of processes, web application. 


\section{INTRODUCCIÓN}

Los resultados de los modelos globales de clima (GCM: Global Climate Models), son las herramientas más avanzadas al momento para evaluar los cambios de patrones del clima futuro, suponiendo la ocurrencia de diversos escenarios de emisiones de gases de efecto invernadero. Generalmente la resolución de los GCMs es muy baja (pixeles de por lo menos 100x100 km), lo cual limita su aplicabilidad para estudios de impacto del cambio climático y su correspondiente efecto en diversos componentes de los ecosistemas, como el ciclo hidrológico (Dibike y Coulibaly, 2005). De allí surge la necesidad de aplicar técnicas de reducción de escala (downscaling en inglés), que permiten aumentar la resolución de las variables climáticas inclusive a nivel de punto o estación.

Las técnicas de reducción de escala se dividen en dos grupos, las técnicas de reducción de escala dinámica y las técnicas de reducción de escala estadística (Fowler y col., 2007). Por un lado, las técnicas de reducción de escala dinámica consisten en aplicar modelos regionales de clima utilizando como condiciones iniciales y de frontera datos de reanálisis o los resultados de los GCMs, y generalmente su aplicación es costosa desde el punto de vista computacional. Por otro lado, las técnicas de reducción de escala estadística buscan relaciones estadísticas entre variables sinópticas y variables observadas de superficie, siendo su aplicación mucho más económica computacionalmente, aunque los mecanismos detrás de esas relaciones sean ocultos al modelador. Debido a la marcada variabilidad espacial y temporal de la precipitación en zonas montañosas como los Andes, al momento las técnicas de reducción de escala dinámicas presentan limitada capacidad predictiva, siento superadas por las técnicas estadísticas, especialmente las que son basadas en técnicas altamente nolineales.

Dentro del estado arte de la generación de escenarios de clima futuros, todavía no existe un acuerdo del valor agregado que los procedimientos de reducción de escala aportan a la estimación de dichos escenarios. Sin embargo, se conoce que los GCMs no representan adecuadamente regiones montañosas, y por lo tanto la representación de los procesos atmosféricos dependientes de la orografía son afectados negativamente, justificando el uso del proceso de reducción de escala. Esto incrementa la incertidumbre existente en la generación de escenarios de clima futuros, que se puede considerar como inherente a: (i) la incertidumbre entre escenarios futuros de emisiones; (ii) la incertidumbre entre modelos GCMs; y (iii) la incertidumbre entre modelos de reducción de escala. Entonces, para la cuantificación de la incertidumbre, desde el punto de vista operacional, se debe incurrir en el manejo de grandes bases de datos para diversos escenarios, para diferentes GCMs, aplicando varios modelos de reducción de escala. Esto hace necesario el desarrollo de una herramienta computacional versátil que facilite el manejo de datos y el procedimiento de reducción de escala. Es así que esta herramienta pueda ser concebida como una parte esencial para el desarrollo de un sistema de soporte de decisiones (DSS) como parte de las medidas no estructurales de adaptación al cambio climático.

Por las razones expuestas, se presenta en este artículo el desarrollo de un sistema con una arquitectura modular basado en el uso de tecnologías web, que encaja en los sistemas DSS actuales. El componente web se integra con herramientas de reducción de escala estadística, específicamente redes neuronales artificiales (ANN) y máquinas de soporte vectorial (SVM), técnicas estadísticas nolineales, de amplia aplicación en ciencias de la tierra. El sistema web en su fase Beta se ha denominado, Statistical Downscaling Workbench (SDW). En la Sección 2 se presenta el procedimiento de reducción de escala y uso de SDW, en la Sección 3 se presenta la arquitectura de la herramienta, mientras que las conclusiones se presentan en la Sección 4.

\section{PROCEDIMIENTO DE REDUCCIÓN DE ESCALA Y USO DE SDW}

El sistema SDW es una aplicación web que surge con la necesidad de crear una herramienta de software que facilite a los investigadores climáticos el hacer reducción de escala usando herramientas computacionales. SDW tiene por objetivo abstraer al usuario de dos tareas complejas y repetitivas asociadas a las simulaciones de reducción de escala: (i) manejo de grandes cantidades de datos 
climáticos que son heterogéneos por naturaleza y provenientes de diferentes fuentes; y (ii) la generación de modelos de reducción de escala y la ejecución de simulaciones de reducción de escala dados modelos previamente calibrados y validados.

\subsection{Datos climáticos necesarios}

Para realizar reducción de escala de GCMs, son necesarias tres fuentes de datos: datos de reanálisis, datos de GCMs y datos de observaciones. Los datos de los GCMs son los resultados de las variables atmosféricas de los modelos globales de clima, mientras que los datos de reanálisis son observaciones de superficie, asimiladas por un modelo climático para generar información en forma de grilla de diversas variables atmosféricas. SDW interpola los datos de grilla al punto donde es necesario aplicar reducción de escala, lo cuál permite la ejecución de reducción de escala en cualquier parte del planeta. Las observaciones son registros generalmente de precipitación o temperatura en una estación.

SDW utiliza un repositorio unificado para la gestión y manejo adecuados de estas tres fuentes de datos. La interfaz gráfica de SDW, para GCMs y reanálisis, posee 3 niveles: (i) Dataset, el nivel superior de organización del conjunto de datos climáticos, (ii) Archivos, ya que usualmente los GCMs o reanálisis se consiguen en varios archivos, y (iii) Variables, que muestra metadata de todas las variables que forman parte de un determinado archivo. En la parte inferior de cada panel existen botones que brindan operaciones básicas de mantenimiento o visualización. Por otro lado, a pesar de que los orígenes y formatos de los datos de observaciones son muy diferentes a los de los de GCMs y reanálisis, son manejados en una organización similar de tres niveles. El nivel superior también tiene una función de agrupación. El segundo nivel es diferente en concepto ya que almacena estaciones, que son puntos geográficos de monitoreo. El tercer nivel, también almacena información relacionada con las variables monitoreadas en una estación. La diferencia operativa fundamental es que los datos de los GCMs y reanálisis se cargan en el segundo nivel usando carga de archivos compatibles (nc, grib, etc.); mientras que, los datos de observaciones se cargan en el tercer nivel usando archivos CSV (Comma Separated Values).

Módulo ECT

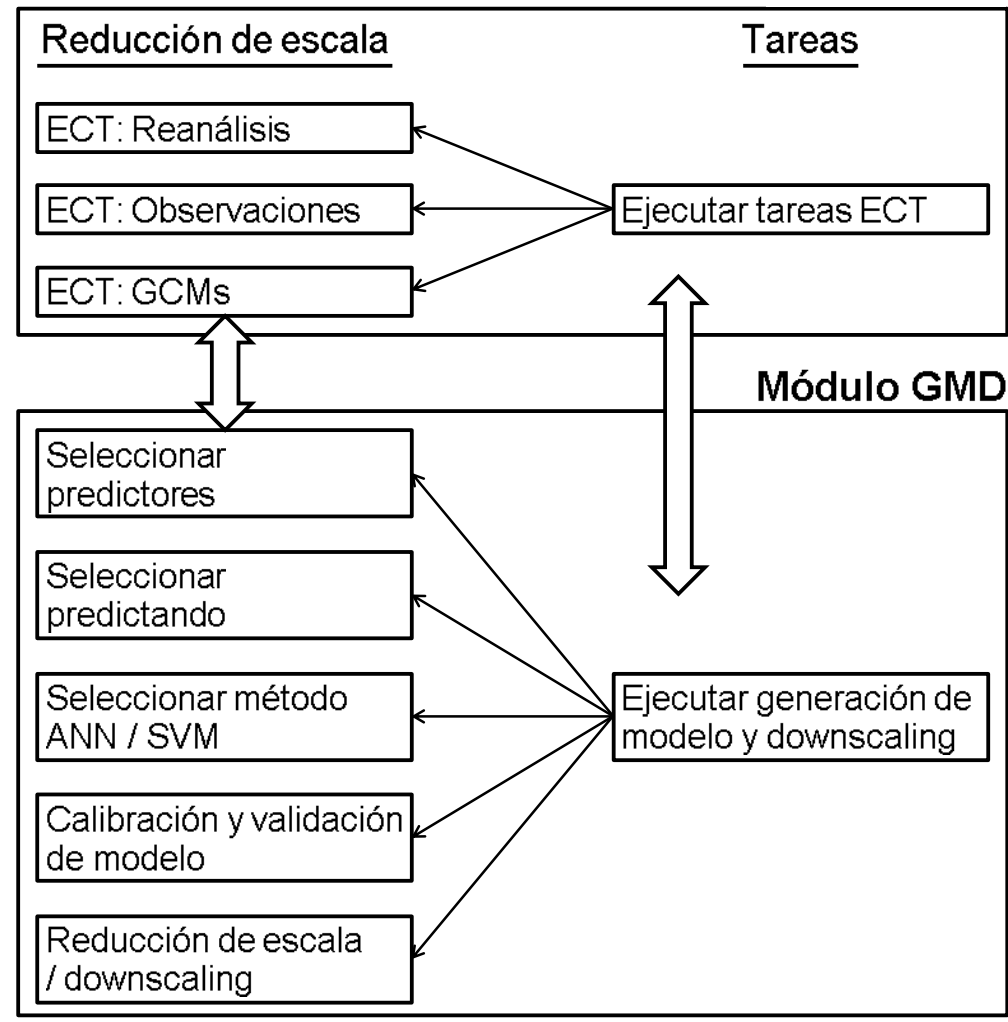

Figura 1. Diagrama de casos de uso de SDW. 


\subsection{Proceso de reducción de escala}

\section{$\underline{\text { Selección de predictores }}$}

El primer paso del flujo de procesos para hacer reducción de escala estadística (Fig. 1), consiste en seleccionar las variables predictoras provenientes de los datos de reanálisis. La variables predictoras son variables relacionadas fenomenológicamente con las variables objetivo, las cuales deben ser determinadas previamente por el usuario. El usuario debe seleccionar el dataset de reanálisis de donde se obtendrán las variables predictoras. Al seleccionar un dataset, todas las variables disponibles para dicho dataset son presentadas automáticamente al usuario. Cada vez que el usuario selecciona una variable, SDW busca automáticamente en el repositorio de GCMs aquellos que son compatibles, es decir, aquellos GCMs que incluyen las variables seleccionadas. De esta forma, el usuario conoce anticipadamente aquellos datasets de GCM que podrá seleccionar para hacer reducción de escala en el presente y futuro. En este punto también se especifican los intervalos de tiempo que se usarán para hacer la calibración, validación y reducción de escala a futuro.

\section{Selección del variable objetivo o predictando}

Una vez seleccionados los predictores, se procede a seleccionar el predictando, que es la variable objetivo para reducción de escala. Para esto, primero se escoge un dataset de observaciones, luego de lo cual automáticamente se cargarán las variables y estaciones asociadas al mismo. El usuario elige la variable que será el predictando y estaciones de interés para el experimento, información que SDW usa para interpolar datos que tenga en forma de grilla.

\section{Selección y configuración del método de reducción de escala}

Una vez definidos los predictores y el predictando, se procede a seleccionar el método de reducción de escala estadístico. Al momento los métodos disponibles son Least Squares Support Vector Machines y Redes Neuronales, aunque la concepción por módulos operacionales de SDW (Fig. 1), permite la ampliación a otros métodos de reducción de escala. Adicionalmente el sistema permite configurar los parámetros para los modelos en forma manual.

\section{Ejecución del método de reducción de escala}

El paso final del flujo de procesos para hacer reducción de escala consiste en ejecutar el método seleccionado previamente sobre los conjuntos de datos extraídos de los predictores y el predictando. En esta etapa se genera el modelo de reducción de escala mediante la calibración de la modelo validada con otro conjunto de datos. La herramienta permite ingresar el número de iteraciones entre las cuales SDW seleccionará el mejor modelo en validación. En esta etapa SDW hace la reducción de escala en el presente, incluyendo los periodos de calibración y validación, usando datos de reanálisis y GCMs, y a futuro solo con datos de GCMs (Cofiño y col., 2007). Además, el usuario puede ver en forma versátil los datos que han sido empleados para la generación del modelo de reducción de escala (series temporales de predictores y predictando), los cuales se muestran en la misma estructura tabular.

\section{ARQUITECTURA DEL SISTEMA SDW}

\subsection{Requerimiento}

Para realizar la compleja tarea de reducción de escala según se indicó en la Sección 2, los requerimientos identifican un único usuario que ejecuta dos grupos de tareas principales del sistema, las cuales han sido agrupadas en dos módulos funcionales (Fig. 1): (i) Tareas ECT (Extracción, carga y transformación) para datos climáticos, que están dentro del módulo ECT; y (ii) tareas de generación del modelo y simulación de reducción de escala estadística (GMD), agrupadas dentro del módulo GMD.

Dentro del primer módulo funcional ECT (Fig. 1), se agrupan las tareas de extracción, transformación y carga (ECT) de datos climáticos provenientes de diversas fuentes. Los datos de 
diversas fuentes en SDW se manejan dentro de un repositorio, el cual organiza los datos climáticos en tres categorías: (i) GCM; (ii) reanálisis; y (iii) observaciones. GCMs y reanálisis tienen estructuras y formatos similares ya que generalmente provienen de archivos con datos científicos orientados a matrices o arreglos donde se almacena una variable por latitud, longitud, niveles y tiempo. Por otro lado, los datos de observaciones pueden tener orígenes y formatos muy variados que dependen de las instituciones responsables. Posiblemente la forma más común en que se comparten los datos de observaciones sea mediante hojas de cálculo o archivos de texto plano. SDW posee una base de datos tanto de reanálisis como de GCMs, sin embargo la herramienta permite cargar datos requeridos por el usuario al repositorio, ya sean de reanálisis, GCMs u observaciones. Por lo tanto el manejo de un repositorio de datos climáticos como parte del sistema SDW ayuda al investigador climático a gestionar en un mismo ambiente los datos y los métodos necesarios para hacer reducción de escala.

El segundo conjunto de tareas corresponde al módulo de generación de modelos y ejecución de reducción de escala estadística, llamado módulo GMD (Fig. 1). Los requerimientos de este módulo definen cuatro etapas sucesivas: (i) selección de predictores; (ii) selección de predictandos; (iii) selección y configuración de modelos de reducción de escala; y (iv) ejecución del método de reducción de escala. Los procedimientos del módulo de GMD usan los datos obtenidos por el módulo ECT.

\subsection{Desarrollo de SDW}

Para el desarrollo de SDW se definieron las siguientes especificaciones como guías para construir una aplicación de calidad, útil y eficiente:

- Crear una interfaz gráfica sencilla y funcional;

- Proporcionar mecanismos para facilitar la integración de herramientas computacionales de inteligencia artificial; y

- Diseñar un flujo genérico para el manejo de las herramientas de reducción de escala estadistica.

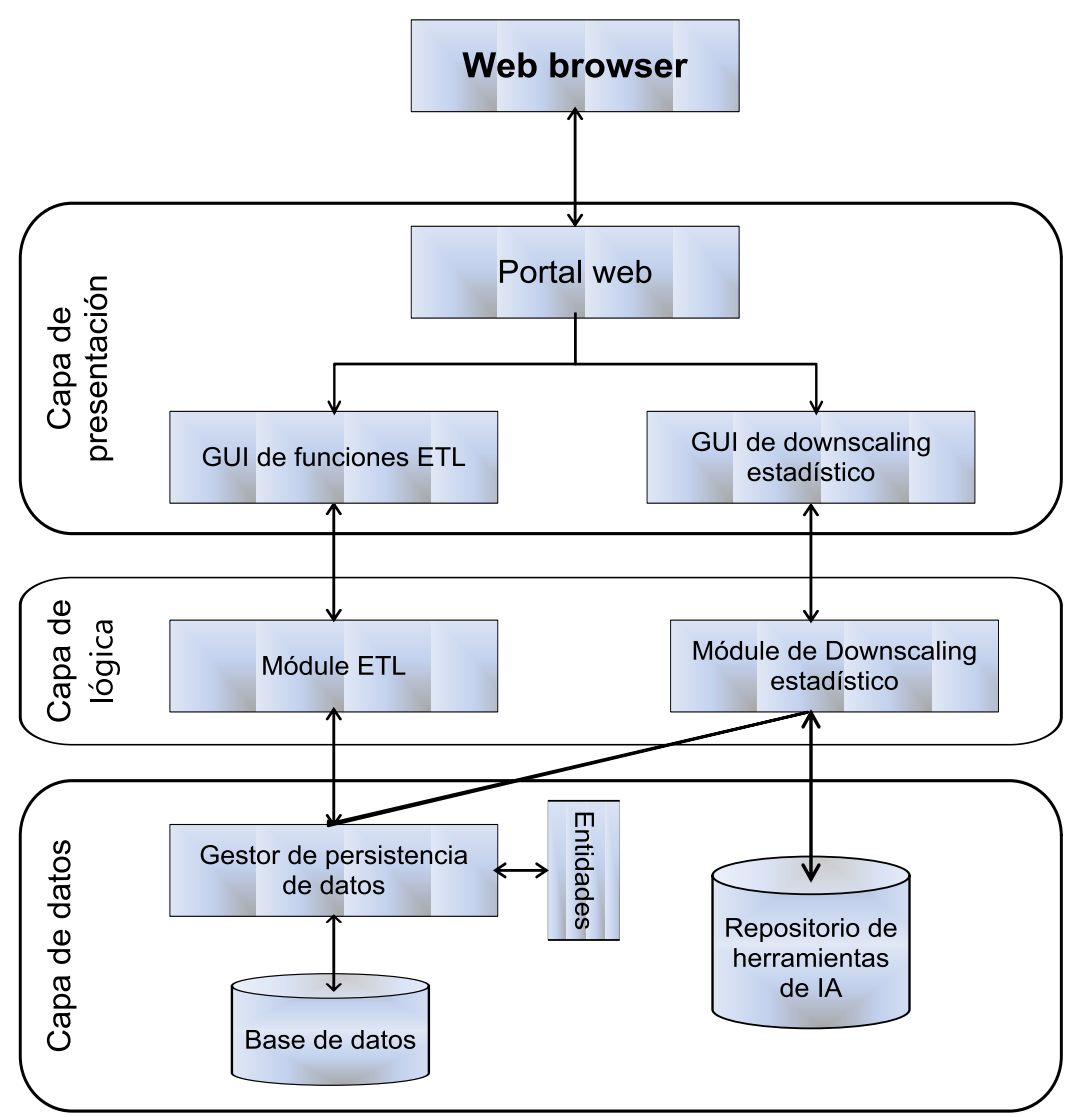

Figura 2. Arquitectura de software. 
A partir del análisis de requerimientos y tomando en cuenta los lineamientos anteriores, se diseñó una arquitectura (Figura 2) basada en la arquitectura de tres capas, que es una arquitectura clienteservidor en la que la interfaz de usuario (capa de presentación), lógica de procesos funcionales (capa de lógica), almacenamiento y accesos de datos (capa de datos), se desarrollan y mantienen en forma de módulos independientes (Eckerson, 1995). Al tratarse de un sistema web, el software único necesario por el usuario final para acceder al sistema es un navegador web. El lenguaje de programación Java fue usado como el principal lenguaje de programación. A continuación se describe con mayor detalle la funcionalidad de cada capa en el contexto del sistema web SDW.

\section{Capa de presentación}

Esta capa contiene las interfaces gráficas del portal web, las cuales se agrupan en dos entornos: (i) repositorio de datos climáticos; y (ii) reducción de escala estadística, correspondientes con los dos módulos funcionales descritos en la sección de Requerimientos. Por consiguiente, el primer entorno proporciona herramientas para cargar datos climáticos desde diferentes fuentes y administrar un repositorio de datos que pueden ser usados para las simulaciones de reducción de escala. El repositorio organiza los datos en tres conjuntos diferentes que internamente son estructurados de forma análoga: datos climáticos de GCM, de reanálisis y de observaciones. Por otro lado, el entorno de reducción de escala estadística brinda un espacio para la generación de modelos y la ejecución de simulaciones de reducción de escala que son tratadas como experimentos. Cada simulación o experimento de reducción de escala consta de 4 etapas básicas: (i) definición del predictor; (ii) definición del predictando; (iii) selección y configuración del método de reducción de escala estadístico; y (iv) ejecución del proceso de reducción de escala. Los datos necesarios para la ejecución de los experimentos de reducción escala son tomados del repositorio de datos climáticos. En la Fig. 3 se muestra una captura de la interfaz gráfica de parte del proceso de reducción de escala, donde se puede ver la representación tabular de los datos que serán empleados para la calibración y validación del modelo de un experimento.

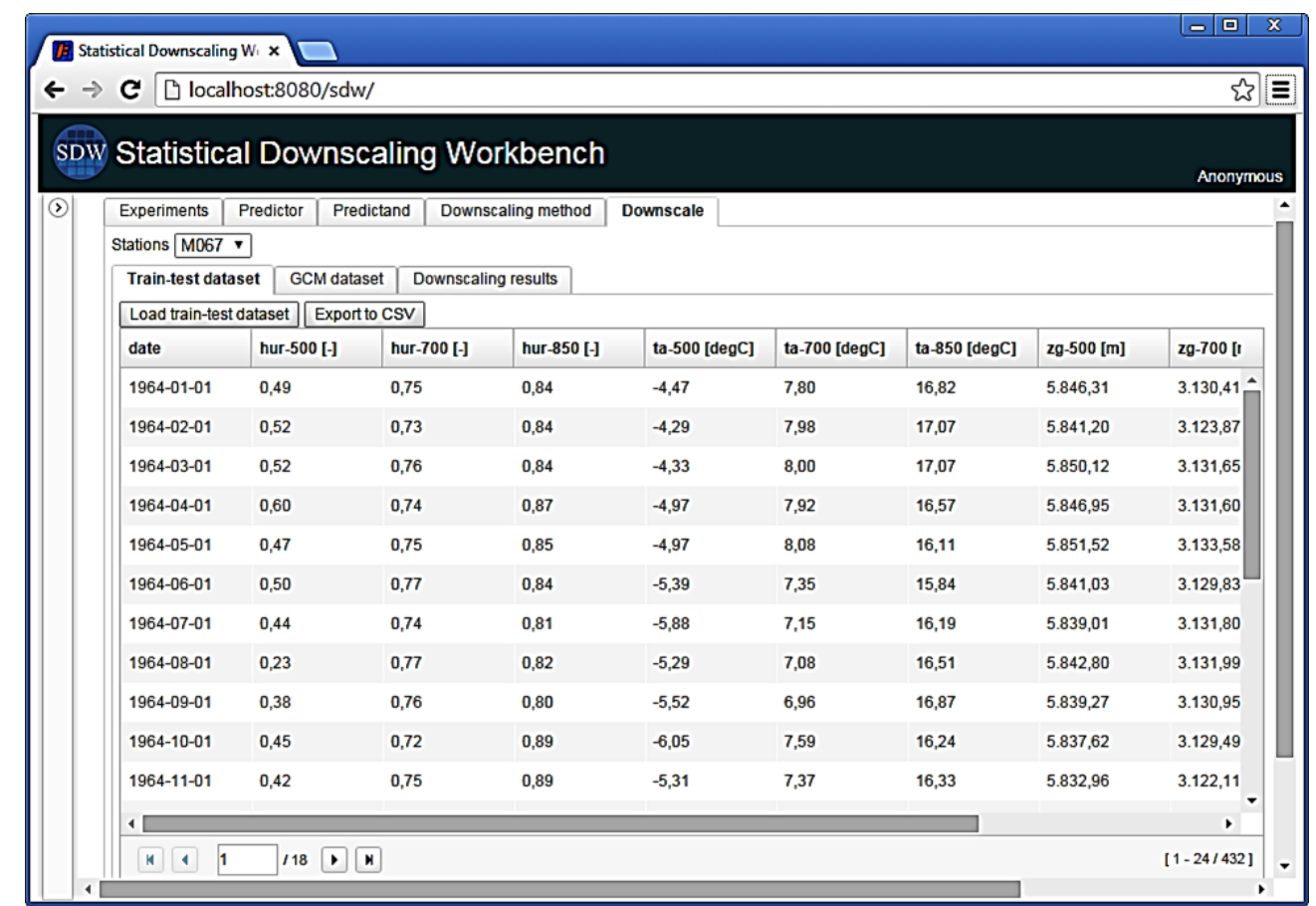

Figura 3. Captura de pantalla de la interfaz gráfica de SDW para la etapa de reducción de escala.

Para el desarrollo de las interfaces de usuario que forman parte de esta capa, se usó el patrón de diseño Model-View-View-Model (MVVM), (Smith, 2009), mediante el uso de la plataforma ZK, que es una plataforma robusta para el desarrollo de aplicaciones web y móviles sin tener que utilizar 
Javascript o AJAX directamente (Zkoss, n.d.). Adicionalmente, se empleó la librería Jfreecharts (JFreeChart, n.d.), para la creación de las representaciones visuales de las series de tiempo de las variables empleadas en las simulaciones de reducción de escala.

\section{Capa de lógica}

Es la capa de procesamiento central responsable de llevar a cabo las tareas principales de la lógica de negocio de SDW. Es decir, esta capa se encarga de la programación de las tareas ECT (Fig. 1) y de las etapas de reducción de escala estadística. Para estructurar esta capa acorde a las mejores prácticas de desarrollo de software empresarial se usó la plataforma Spring, que proporciona un modelo de programación y configuración completa para las aplicaciones empresariales modernas basadas en Java (Spring Framework, n.d.). De esta forma, se logró estructurar ordenadamente los vínculos entre las tres capas de la arquitectura, permitiendo profundizar el esfuerzo de desarrollo en la lógica de negocio de la aplicación. Para el desarrollo del módulo ECT se usó la librería NetCDF-Java, que es una interfaz Java para archivos NetCDF, así como para muchos otros tipos de formatos de datos científicos. NetCDF implementa un Modelo Común de Datos (CDM), que es una generalización de los modelos de datos NetCDF, OPeNDAP y HDF5, que permite trabajar con varios tipos de archivo como netCDF, GRIB, HDF, OPeNDAP, etc. (Unidata, n.d.). A su vez, para el módulo GMD, se usó el patrón de diseño adaptador (Freeman y col., 2004), que permiten estandarizar el manejo de las herramientas computacionales de reducción de escala, ya que acorde a los lineamientos de desarrollo SDW debe facilitar y promover la integración de herramientas computacionales de reducción de escala independientemente de la naturaleza o implementación de dichas herramientas.

\section{Capa de datos}

Es la capa responsable del manejo de la base de datos mediante el uso de técnicas de persistencia con JPA (Java Persistence API), que es una plataforma que cubre la brecha existente entre los modelos del dominio orientado a objetos y los sistemas de base de datos relacionales (Keith y Schincariol, 2009). Además, contiene un repositorio de modelos que alberga los ejecutables de las técnicas de inteligencia artificial para la ejecución de métodos de reducción de escala estadístico.

\subsection{Despliegue e implementación}

Gracias a la estructura en capas del sistema SDW, el despliegue del sistema se puede hacer en un solo computador. La arquitectura física del sistema se muestra en la Fig. 4. Dentro del servidor central la comunicación con los ejecutables de los modelos de reducción de escala se realiza por medio de

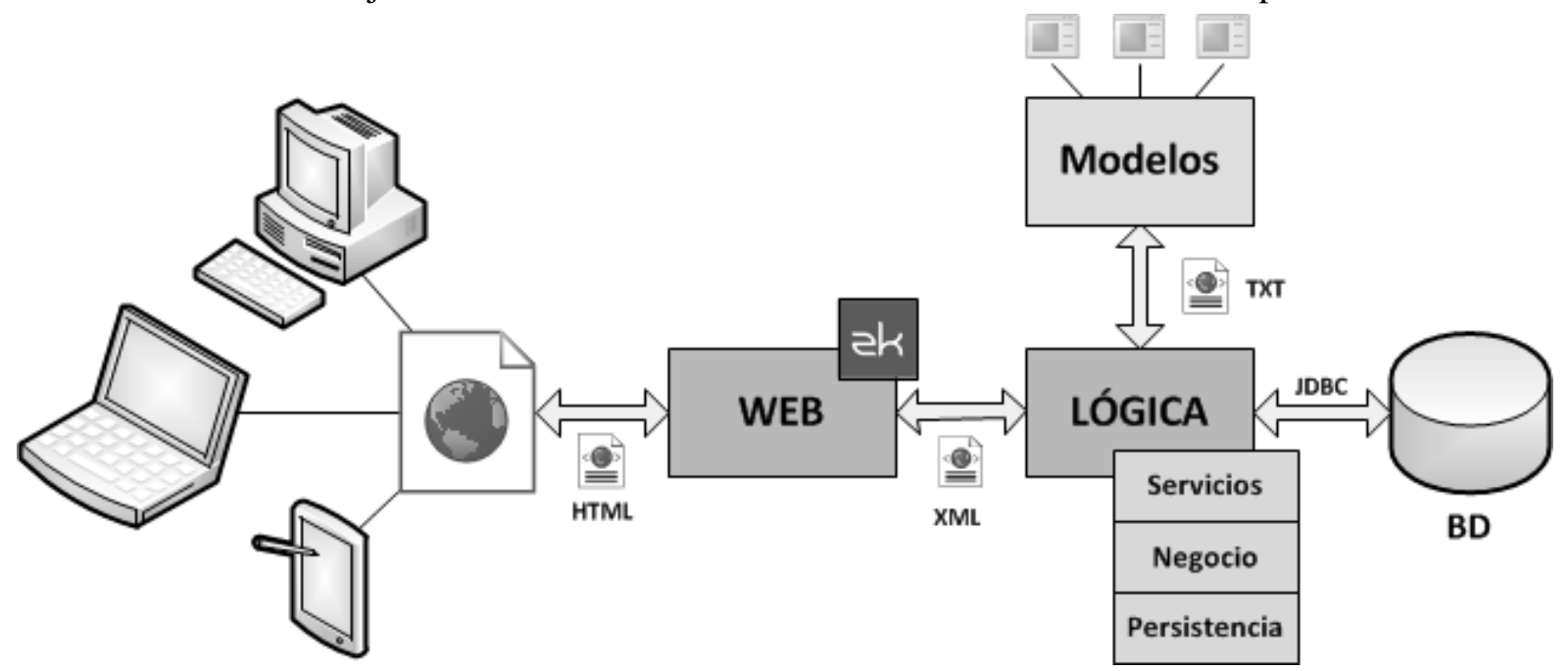

Figura 4. Arquitectura física de SDW. 
archivos de texto plano o llamadas directas. Esto se debe a que algunos de los ejecutables de las técnicas de inteligencia artificial leen los datos de entrada desde un archivo de texto plano y similarmente los resultados son redirigidos hacia archivos también de texto plano.

\section{CONCLUSIONES}

Se ha presentado el desarrollo del sistema denominado Statistical Downscaling Workbench (SDW), un sistema web en versión Beta, que tiene como objetivo permitir a los investigadores climáticos ejecutar simulaciones de reducción de escala de los modelos globales de clima, usando dos técnicas de inteligencia artificial, ANN y LS-SVM, a fin de obtener información climática futura de alta resolución espacial que pueda ser empleada para estudios de impacto de cambio climático.

SDW agrupa su funcionalidad en dos módulos o subsistemas. El módulo de extracción, carga y transformación, ECT, permite almacenar en un repositorio relacional, datos provenientes de archivos de reanálisis, GCMs y de observaciones. El módulo de generación de modelos y reducción de escala, GMD, permite ejecutar las diferentes etapas necesarias para la ejecución de técnicas de reducción de escala estadístico. Para el diseño se definió una arquitectura en capas, que de cara al usuario presenta un portal web. Como uno de los lineamientos para el desarrollo del sistema se definió la creación de un flujo de procesos genérico que permita utilizar los modelos de manera unificada. Esto permite y facilita la integración de nuevos modelos a futuro, característica que suma versatilidad y utilidad a esta herramienta, que formaría una parte fundamental dentro de un sistema de soporte de decisiones para la adaptación al cambio climático.

\section{AGRADECIMIENTOS}

Este trabajo ha sido financiado por la Dirección de Investigación de la Universidad de Cuenca (DIUC) a través del proyecto "Análisis de los Efectos del Cambio Climático en los caudales en las Cuencas Andinas del Sur del Ecuador (Paute), debido a los Cambios en los patrones de lluvia y Temperatura".

\section{REFERENCIAS}

Cofiño, A.S., D. San-Martin, J.M. Gutiérrez, 2007. A Web portal for regional projection of weather forecast using GRID middleware. In: Shi, Y. et al. (Eds.): ICCS 2007, Part III, LNCS 4489, 8 pp. Springer-Verlag Berlin Heidelberg. Disponible en http://www.meteo.unican.es/files/pdfs/2007_ gutierrez_LNCS.pdf.

Dibike Y.B., P. Coulibaly, 2005. Hydrologic impact of climate change in the Saguenay Watershed: Comparison of Downscaling Methods and Hydrologic Models, J. Hydrol., 307(1-4), 145-163.

Eckerson, W.W., 1995. Three tier client/server architecture: Achieving scalability, performance, and efficiency in client server applications. In: Conference Open Information Systems 10, 3-20.

Fowler, H.J., S. Blenkinsop, C. Tebaldi, 2007. Linking climate change modelling to impacts studies: Recent advances in downscaling techniques for hydrological modelling. Int. J. Climatol., 27(12), 1547-1578.

Freeman, E., B. Bates, K. Sierra, 2004. Head first design patterns. Paperback, O'Reilly Media, Inc.

JFreeChart [WWW Document], n.d. Descargad de http://www.jfree.org/jfreechart/ el 30 de agosto de 2014.

Keith, M., M. Schincariol, 2009. Mastering the Java ${ }^{\mathrm{TM}}$ persistence API. APRESS, $538 \mathrm{pp}$. Disponible en http://www.dainf.ct.utfpr.edu.br/ caio/hibernate/pdf/Apress\%20-\%20Pro\%20JPA\%202\%20 Mastering\%20the\%20Java\%20Persistence\%20API\%20(November\%202009)\%20(ATTiCA).pdf. 
Smith, J., 2009. WPF Apps with the Model-View-ViewModel (MVVM) design pattern. MSDN Magazine. Disponible en http://msdn.microsoft.com/en-us/magazine/dd419663.aspx.

Spring Framework [WWW Document], n.d. Descargado de http://projects.spring.io/springframework/ el 30 de agosto de 2014.

Unidata, NetCDF [WWW Document], n.d. Unidata NetCDF. Descargado de http://www.unidata. ucar.edu/software/netcdf/ el 21 de marzo de 2012.

Zkoss, n.d. Leading Enterprise Java Web Framework | ZK [WWW Document]. Descargado de http://www.zkoss.org/el 29 de enero de 2013. 